\title{
Improving Mental Status Questionnaire (MSQ) completion on admission to the Acute Surgical Receiving Unit (ASRU), Ninewells Hospital, Dundee
}

\author{
Sylvia Okwemba, Lauren Copeland
}

NHS Tayside

\begin{abstract}
Delirium is common yet poorly identified in the UK. Early recognition is a key prognostic factor; delay here being associated with: increased mortality, increased morbidity, prolonged hospital stay, long term disability, and increased risk of developing dementia. Improvement in the diagnosis and management of delirium has scope to improve patient care, clinical outcomes, and ultimately an improved patient experience. As patients aged $\geq 75$ years are at an increased risk of developing delirium, we focused the improvement project to this age group.
\end{abstract}

The baseline data demonstrated that the average $\geq 75$ year-old patient admitted to the Acute Surgical Receiving Unit (ASRU) at Ninewells Hospital had 5.4 out of 12 predisposing and precipitating risk factors for delirium; thus there was great potential for delirium to develop in these patients. During the analysis of the baseline data it became clear that we could not go ahead and implement the initial proposed improvement as the completion of the mental status questionnaire (MSQ) was inconsistent and low at $14.99 \%$. Completion of the MSQ is vital in establishing any cognitive deficit at admission, and for providing a baseline for the continuing admission. As a consequence of this, we had to shift the main aim of the improvement project from improving the identification, diagnosis, and management of delirium, to improving the completion rate of the MSQ in our target age group.

Consultations with members of the admission team were held to determine ways of improving the MSQ completion rate. It became clear that the completion of the MSQ relied on clinical staff remembering all 10 questions that constitute the test. The main intervention to facilitate improvement involved affixing a sticker with all 10 questions of the MSQ within the admissions document. The main aim was to increase the percentage of cognitive screening by the Mental State Questionnaire (MSQ) to $95 \%$ in patients aged $\geq 75$ on admission to ASRU at Ninewells Hospital by 11 th July 2013.

We achieved our main aim with $100 \%$ compliance on two days. Our average compliance over six days was however $81.33 \%$, whilst not reaching our target this is still a substantial improvement. The introduction of the sticker detailing the 10 MSQ questions within the ASRU admissions document was well received by the admissions team. It has simplified the process as members of staff do not need to rely on their memory to remember the questions, and the sticker also acts as a prompt for them to consider further cognitive screening.

\section{Problem}

Delirium is common yet poorly identified in the UK (1) with NHS Scotland making the identification and management of patients with delirium a national priority. Healthcare Improvement Scotland via "Improving Care for Older People in Acute Care" has been involved in running educational work within NHS Scotland to try and address the burden associated with the poor recognition, investigation, and management of delirium. Early recognition is a key prognostic factor; delay here being associated with: increased mortality, increased morbidity, prolonged hospital stay, long term disability, and increased risk of developing dementia (2-5). Ninewells Hospital is based in the outskirts of Dundee in the East of Scotland, and caters for a wide number of areas in Angus. It is an acute care tertiary centre and contains 862 staffed beds with a full range of healthcare specialties.

\section{Background}

At a local level, a recent review of case notes within the Acute
Medical Unit (AMU) of Ninewells Hospital provided an early indication that delirium is poorly recognised in a significant proportion of the patients considered. Improvement in the diagnosis and management of delirium has scope to improve patient care, clinical outcomes, and ultimately generate an improved patient experience. Finally, from an organisational perspective, early recognition should feasibly decrease costs in the long-term.

\section{Baseline measurement}

Prior to the collection of baseline data, a data collection tool was created to enable us to capture sufficient information to understand current clinical practice in ASRU. Our inclusion criteria for the collection of baseline data included: patients aged 75 years or over and patients admitted within a specified 24 hour period. The exclusion criteria included: patients $<75$ years of age, patients who are discharged or transferred to another hospital, and patients who died during their admission. The admission notes of 39 patients $\geq 75$ years of age during a 10 day period were examined to elicit: the presence of common predisposing and precipitating factors of delirium, their mental status by documentation of the Mental Status 


\section{BMJ Quality Improvement Reports}

Questionnaire (MSQ) score and any stated descriptors of mental state, and the presence of a diagnosis of delirium, its subsequent investigation and management. Common risks and precipitating factors for delirium include: infection, recent medication change, metabolic disturbance, polypharmacy, multiple chronic conditions, hearing or visual impairment, cognitive impairment, age $>75$, and physical restraint, e.g. IV lines or catheters (6).

For all of the patients, their age, sex, presenting complaint, and admissions diagnosis was documented. No patient had a formal diagnosis of delirium, however most patients had precipitating factors for delirium with the range being 2-10 out of a total of 12 factors. The mean number of precipitating factors for delirium in the average $\geq 75$ years old ASRU patient was 5.4 , demonstrating that there was a great potential for delirium to develop in these patients.

See supplementary file: ds3425.docx - "Version 11 - Data Collection Tool"

\section{Design}

During the analysis of the baseline data, it became clear that we could not go ahead and implement a change to facilitate the identification of delirium as the MSQ completion rate was inconsistent and low at $14.99 \%$. To further explore this issue, we assessed the MSQ score for the patients included in the baseline data. Most of the scores fell between a score of 5-10 out a total of 10. According to local clinical guidelines, patients with an $M S Q$ score of 7 or less require prompt re-evaluation by a senior clinician.

When considering the underlying cause of this problem, it became clear that the completion of the MSQ score on the admissions document relied on the clinical staff remembering all 10 questions that constitute the MSQ, as these questions were not included within the document. During research into ways of improving cognitive screening on admission in our target group, we came across an admissions document from a similar acute setting (AMU) that had detailed the $10 \mathrm{MSQ}$ questions that were missing from the surgical admissions document. The main intervention would involve affixing a sticker with all 10 questions of the MSQ within the admissions document. The sticker was to act both as a prompt for the admitting clinician to remember to do the MSQ, and also to facilitate easy completion of the MSQ.

The plan was to initially introduce a sticker detailing the $10 \mathrm{MSQ}$ questions to one member of the admissions team with the aim of allowing them to give feedback on its usability. Based on this feedback we had planned to then expand our test of change via inclusion of the sticker within the clerking document to be used by all members of the admission team on our target patient group. The main aim was to increase the percentage of cognitive screening by the Mental State Questionnaire (MSQ) to $95 \%$ in patients aged $\geq 75$ on admission to the Acute Surgical Admissions Unit at Ninewells Hospital by 11 th July 2013.

\section{Strategy}

PDSA 1

The objective of our first PDSA cycle was to develop a tool to enable the collection of baseline data demonstrating the current identification, diagnosis, and management of delirium. We aimed to test the efficiency, usability, relevance, and reliability of the data collection tool within ASRU at Ninewells Hospital, Dundee. Due to limited access to patient notes, we needed to ensure that the tool could be completed accurately in good time ( $<10$ minutes). The usability and reliability of the tool was important as it could be used in the future as a measure of improvement and it should be accessible to all members of staff regardless of training. The test would be performed on one patient initially; with the number of patients involved gradually being increased until we were satisfied that the tool fulfilled the parameters stated above.

The information we wanted to collect during this PDSA cycle included: the amount of time it took to fill in the tool, whether the tool could be filled in by any member of staff without prior training, whether the tool was sensitive enough to pick up the major precipitating and contributory factors, clinical features, diagnosis and management of delirium and whether different team members arrived at the same conclusion when filling the form in independently.

Whilst trialling our data collection tool, we observed that the clinical presentation for potential patients with delirium was often described in colloquial terms e.g. "off their legs". We also observed that some of the major precipitating/contributory factors and clinical features recurred in patients but were missing from the tool (e.g. metabolic disturbance and hearing/visual impairment). The wording in certain sections of the first draft of the tool was ambiguous and compromised the usability of the form. We then had to tweak the original tool and develop multiple new drafts to overcome our observations. Initially, the completion of the tool took 22 minutes. We improved the general flow of the tool to compliment the layout of the patient notes; this dramatically improved the ease of obtaining relevant data. We removed the ambiguity of the language and also implemented a "tick box" format to reduce the writing load. This reduced the total time required to fill in the tool to 3.5 minutes.

Upon analysing literature around the subject, we decided to include a more exhaustive list of the precipitating/contributory factors for delirium in the tool. We also forwarded drafts of the tool to our clinical contacts with expertise on the topic to obtain more advice on the content of the tool. Initially, the diagnosis and management sections of the tool were integrated, but we later separated the two sections to enable us to collect more data on the management of delirium whilst maintaining clarity. Separating the diagnosis and management of delirium sections also enabled us to disregard the management section if the diagnosis of delirium was not made, increasing the relevance of the information collected. By the time we had a tool that ticked all the parameters we had set out, we had drafted a total of 11 tools. Using our completed tool, we were then able to collect baseline data on the identification, diagnosis and management of delirium in ASRU. 
The baseline data obtained from the first PDSA cycle clearly demonstrated that there was poor compliance amongst staff with completion of cognitive screening (MSQ) on patients aged 75 years and over on admission despite this being part of the admission documentation. During the first five day period of data collection, completion of the MSQ in the above age-group was $16.6 \%$ and in the second five day period, this dipped further to $13.3 \%$. From this data, it was evident that there was no consistent practice in place to facilitate identification of cognitive impairment upon admission and as such, without the baseline information on a patient's cognitive state, we decided that it would be unrealistic to introduce a screening tool to identify delirium at this stage.

The main objective of the second PDSA cycle was therefore to improve the percentage of $\mathrm{MSQ}$ assessments completed for the target patient group. Before we could identify a change to implement, we wanted to gain a greater understanding of reasons for the low compliance rate amongst the admissions staff team (junior doctors and advanced nurse practitioners - ANPs). We created a questionnaire and used this in the ward environment to capture data to answer the above question. Furthermore, we looked to other ward environments within Ninewells Hospital for inspiration. Using this qualitative data, we were able to identify an area for change. The feedback from staff centered around a lack of knowledge of the questions to use as these themselves were not included within the admissions document.

This PDSA cycle was focused on testing the addition of the MSQ questions as a sticker within the admissions document. The hope was that the sticker detailing the 10 questions would act as a prompt or aide-memoire to address the apparent knowledge deficit on the $10 \mathrm{MSQ}$ questions. The sticker was first introduced to one member of the admissions team and with the aim of them providing feedback on its usability. Based on this feedback we hoped to expand our test of change via inclusion of the sticker within the admissions document to be used by all members of the admission team on our target patient group. Once that was done, we would continuously monitor the process utilising the data collection tool we developed in PDSA \#1.

The information we aimed to collect included: the number of MSQs completed for patients aged 75 years and over on admission and qualitative data utilising a questionnaire to determine the number of staff trained on performing the MSQ, general awareness of the rationale behind the MSQ and how this relates to delirium. We also wanted to collect staff feedback on: the time it takes to complete the $M S Q$, whether completing the MSQ affected other aspects of the admission process and general staff satisfaction relating to this change.

When we initially implemented the change, we found that it was difficult to engage all staff involved in the admissions process due to the rapid turnover of staff, especially junior doctors who rotated around other surgical wards on a weekly basis. The ANPs were the most consistent team members of the admissions team and therefore we encouraged them to advise new junior doctors of the change at each new shift rotation. We also e-mailed all the junior doctors to advise them of the work we were doing to ensure that they were fully aware of what was happening.

Our first prediction was that the inclusion of the sticker would improve percentage compliance of completion of the MSQ on admission in our target group. Overall, this predication was accurate as compliance with MSQ completion increased following the introduction of the measure. The average MSQ completion rate was $13.3 \%$ during our baseline collection period. During the 10 day period, the completion of the MSQ was random and not consistently performed with some $0 \%$ points. Once we introduced the sticker, we audited the process over a period of 6 days. We did this to ensure that we captured data during the weekend as this is known to be a problematic period for numerous reasons e.g. fewer nursing and medical staff cover. The average MSQ completion rate after the introduction of the MSQ stickers was $81.33 \%$. This is a good improvement as we have a shift with most of our data points being above the median line.

Feedback from the admissions staff informed us that the time taken to complete the admissions process was generally not adversely affected due to the brevity of the MSQ test. In addition, other aspects of the admissions process e.g. examination of the patient and sufficient history taking were not compromised.

We had predicted that due to frequent medical staff rotations and no clear medical leadership in the ward environment, change may be slow to develop. This prediction was accurate. On the first day of data collection after inclusion of the MSQ stickers, 3 July 2013, the compliance was $40 \%$. Whilst we recognise this as an improvement, it was not as significant as we had initially hoped for. At this point we had an informal discussion with the admissions team and it became obvious that not all relevant people were aware of the change implemented due to recent staff rotation. On the 5th of July 2013, we achieved $100 \%$ compliance with the MSQ and we attribute this success to clear signage within the admissions bay and input from senior nursing team members who acted to disperse the message and remind staff. Unfortunately, the compliance was then observed to dip over the weekend, 6th \& 7th July 2013, and we realised that awareness may still be a problem amongst the weekend staff. Such frequent staff rotations make improvement difficult to sustain and ultimately in order to tackle this, it may be prudent to introduce the work being done around the MSQ during a staff member's induction to the surgical ward to ensure this is at the forefront of their mind. Our target was 95\% completion of the MSQ on all new admissions in our target group, we achieved this with $100 \%$ compliance on two days. Our average compliance was $81.33 \%$, whilst not reaching our target this is still a substantial improvement.

\section{Results}

Overall, the introduction of the MSQ sticker to the clerking document resulted in a significant improvement in the percentage of MSQs that are completed on patients aged 75 and older upon admission to the Acute Surgical Receiving Unit (ASRU). Previously, the compliance with the MSQ was inconsistent with several $0 \%$ points as well as astronomical points as illustrated on the run chart. A run of 5 points above the median can be seen since the 
implementation of the sticker indicating that improvement has occurred. We initially introduced the sticker to one member of staff and then by affixing this to the clerking document, rolled the change out to all members of the admissions team - advanced nurse practitioners and junior doctors. A high turnover of junior medical staff meant it was challenging to ensure everyone was aware of the change. We feel this could be addressed via information given at a ward induction for new members of staff.

We had predicted that the implementation of the MSQ sticker would allow us to reach our goal of $95 \%$ compliance with completing the MSQ on all new admissions in the target age group. We achieved this with $100 \%$ compliance on two days. Our average compliance rate was $81.33 \%$ in comparison to $14.99 \%$ from the previous 10 day period during which we collected our baseline data. Whilst not reaching our target, this is still a substantial improvement. There is a noticeable difference in compliance levels over the weekend and by further investigating reasons for this - most likely, a lack of awareness coupled with lower staffing levels - the average compliance could feasibly improve further. Furthermore, having the MSQ questions permanently affixed to the document could sustain an increase in compliance in the long-term as the stickers used currently may allude to a temporary change.

The introduction of the MSQ stickers has resulted in an improvement within the ASRU on cognitive screening on admission in our target group. We strongly feel that continuously speaking to the admissions team and junior doctors about the project has definitely increased awareness on cognitive screening. These improvements are encouraging as they bring ASRU in line with similar acute clinical environments within Ninewells Hospital i.e. the Acute Medical Admissions Unit (AMU) who already had the MSQ questions within their clerking document and are achieving similar levels of completion.

See supplementary file: ds3672.pdf - "ASRU Run Chart Demmonstrating the Impact of Improvement Interventions"

\section{Lessons and limitations}

On reflection, the success of the project is largely attributed to the willingness of the admissions team to take on the aims of the project. In particular, the compliance of the advanced nurse practitioners who are the most permanent of the staff has provided a strong backbone to the improvement process. They have encouraged the junior medical staff and thus helped address the issues surrounding frequent staff rotation. Furthermore, in maintaining contact with the staff and making them aware of the reaudit process throughout, we feel it has been easier to keep the project at the forefront of people's minds.

One of the main limitations of this project is the lack of additional data to demonstrate whether the improvement that was achieved during this project has been maintained by ASRU. The effect of the intervention could only be measured over a 6-day period and therefore additional data points are currently not available. However, as the project was part of a much bigger project, we feel that the work we did significantly informed further work that the
Older People in Acute Care Collaborative subsequently carried out.

Teamwork has been an important theme of the project both in the sense of teamwork between ourselves, the learners in order to drive the improvement process and also as discussed above, amongst the admission staff. In addition, the support we have received from academic staff and those with clinical expertise has been invaluable. Without this, we feel the project would almost certainly not have been as successful. Barriers to success were: the constant rotation of junior staff, no point of contact/ leadership from senior medical staff and the lack of clarity regarding a clinical management pathway for those with low MSQ scores. We have learnt that behavior change is a multi-factorial process and whilst we have seen success in the short-term with our project, in order for this change to be sustainable in the future it will be important to consider the barriers mentioned and further address improvement within the "system" of ASRU at different levels.

\section{Conclusion}

The introduction of the sticker detailing the $10 \mathrm{MSQ}$ questions within the ASRU admissions document was well received by the admissions team and has led to significant improvement without adding a step into their current admissions process. It has simplified this process as members of staff do not need to rely on their memory to remember the questions and the sticker also acts as a prompt for them to consider cognitive screening.

As this project is part of the larger Older People in Acute Care Collaborative (OPACC), we feel that the work we have done can be added upon to achieve sustainability in the long term. The training of all members of staff of the admissions team on the MSQ and its relevance in respect to cognitive impairment will further support the intervention we accomplished during this project. Buy in from senior clinical physicians is vital as they can provide valuable input into the clinical management pathway for patients with clinically relevant MSQ scores. We aim to provide feedback to the ASRU admissions team on the improvement that has occurred so far and will advise them to constantly re-audit the process to ensure compliance is maintained.

\section{References}

1. National Institute of Clinical Excellence (2010). Delirium. Diagnosis, prevention and management. Available from: http://www.nice.org.uk/nicemedia/live/13060/49909/49909.p df

2. O'Keefe S, Lanvan J (1997). The prognostic significance of delirium in older hospital patients. Journal of the American Geriatrics Society; 45(2):174-178

3. Siddiqui N, O'House A, Holmes JD(2006). Occurrence and outcome of delirium in medical in-patients: a systematic literature review. Age and Ageing; 35(4):350-364

4. van den Boogaard M, Schoohoven L, Evers AW, van der Hoeven JG, van Achterberg T et al(2012). Delirium and critically ill patients: impact on long-term health-related quality of life and cognitive functioning. Critical Care 


\section{BMJ Quality Improvement Reports}

Medicine;40(1):112-118

5. McCusker J, Cole M, Dendukuri N, Belzile E, Primeau $F(2001)$. Delirium in older medical inpatients and subsequent cognitive and functional status: a prospective study. Canadian Medical Association Journal;165(5):575-583

6. Gesin G, Russell BB, Lin AP, Notron J, Evena SL, Devlin JW (2012). Impact of a Delirium Screening Tool and Multifaceted Education on Nurses' Knowledge of Delirium and Ability to Evaluate It Correctly. American Journal of Critical Care; $21(1): 1-11$

\section{Declaration of interests}

None

\section{Acknowledgements}

Junior Doctors, Nurses \& Allied Health Professionals of Ward 7 (ASRU)

Prof P. Davey, Medical School Lead for Clinical Quality Improvement, University of Dundee.

Vicki Tully, Teaching Lead for Patient Safety, University of Dundee Medical School.

Marie Hanlin, Older Peoples' Collaborative

Morag McRae, Patient Safety Development Manager

Alison Davie, NHS Tayside Improvement Advisor - Patient Safety

Student Improvement Team - Yevedzo Ntuli, Kirsty McNeil, Jane

Vennard-Balmer, Claire Pollington \& Avril Anderson 